\title{
RED BAYESIANA PARA ANALIZAR LOS MECANISMOS DE ADAPTACIÓN DE LOS COMERCIANTES ANTE LOS RETOS DE LA COVID-19
}

BAYESIAN NET TO ANALYZE THE MECHANISMS OF ADAPTATION OF THE MERCHANTS IN THE PRESENCE OF THE CHALLENGES OF THE COVID-19

Gloria Pascuala Chiquito Tigua

Docente, Universidad Estatal del Sur de Manabí, (Ecuador). E-mail: g_chiquito@hotmail.com ORCID: https://orcid.org/0000-0002-3593-2049

Ninieve Victoria Plua Parrales

Docente, Universidad Estatal del Sur de Manabí, (Ecuador). E-mail: abvickplua_p@hotmail.com ORCID: https://orcid.org/0000-0001-5824-9755

Jessenia Herminia Morán Chilán

Docente, Universidad Estatal del Sur de Manabí, (Ecuador). E-mail: jessenia.moran@unesum.edu.ec ORCID: https://orcid.org/0000-0002-3853-6017

\section{Citación sugerida:}

Chiquito, G. P., Plua, N. V., y Morán, J. H. (2020). Red Bayesiana para analizar los mecanismos de adaptación de los comerciantes ante los retos de la COVID-19.3CEmpresa. Investigación y pensamiento crítico. Edición Especial COVID-19: Empresa, China y Geopolítica, 67-81. https://doi.org/10.17993/3cemp.2020. edicionespeciall.67-81 


\section{RESUMEN}

Los mecanismos de adaptación de los comerciantes ecuatorianos, ante los retos de la COVID-19, constituyen elementos a tener en cuenta para el desarrollo económico post pandemia. Para enfrentar la COVID-19, los gobiernos han impuesto las acciones del autoaislamiento, la cuarentena y el distanciamiento social, medidas importantes para enfrentar la COVID-19 y contener la expansión del virus. Estas medidas tienen impactos positivos para la salud, al aplanar la curva de contagio, pero, afecta la actividad económica, pues el distanciamiento, generalmente implica la desaceleración de la producción o incluso su interrupción total, ya que se disminuyen las horas de trabajo y los salarios, factores que ocasionan una reducción de la demanda agregada de bienes y servicios. Por tal motivo, en el presente trabajo se realiza un análisis de sensibilidad a la evidencia en Redes Bayesianas, para analizar los mecanismos de adaptación, de los comerciantes, ante los retos de la COVID-19, análisis útil para verificar la volatilidad de los mercados financieros relativos a los comerciantes ecuatorianos, en particular, como resultado de la incertidumbre respecto de la intensidad y la duración de la pandemia, la paralización económica y el impacto de las exportaciones en la región, cuyo volumen y valor se ha reducido significativamente debido a la recesión mundial causada por la COVID-19.

\section{PALABRAS CLAVE}

Análisis de sensibilidad, Red bayesiana, Mecanismos de adaptación de los comerciantes, COVID-19, Pandemia. 


\section{ABSTRACT}

The mechanisms of adaptation of the Ecuadoran merchants, in the presence of the challenges of the COVID-19, constitute elements to keep in mind for the economic development pandemic post. To face the COVID-19, the governments have imposed the actions of the self-isolation, the forty and the social, measured important distancing to face the COVID-19 and contain the expansion of the virus. These measures have positive impacts for the health, when smoothing the curve of contagion, but, affectionate the economic activity, because the distancing, generally implies contradiction the deceleration of the production or even your total interruption, since decrease to him the hours of work and the salaries, factors that occasion a reduction of the added claim of properties and services. For such motive, at present work an analysis of sensibility to the evidence in Bayesian Net is carried out, to analyze the mechanisms of adaptation, of the merchants, in the presence of the challenges of the COVID-19 useful analysis to verify the volatility of the financial relative markets to the Ecuadoran merchants, specially, as a result of the uncertainty concern of the intensity and the duration of the pandemic, the economic stagnation and the impact of the exports in the region, whose volume and value been reduced significantly due to the worldwide recessions caused for the COVID-19.

\section{KEYWORDS}

Analysis of sensibility, Bayesian net, Mechanisms of adaptation of the merchants, COVID-19, Pandemic. 


\section{INTRODUCCIÓN}

La COVID-19 es una enfermedad originada por el virus SARS-GoV-2, que ha azotado países y continentes, ha causado un nivel inusitado de sufrimiento humano, agitación social y daño económico, la expansión de la crisis actual tiene precedentes en los comerciantes y en particular en los comerciantes ecuatorianos. La economía en Ecuador vive una crisis sanitaria, humana y económica sin precedentes, que evoluciona continuamente junto a la pandemia, las economías se cierran y paralizan las sociedades al entrar en cuarentenas más o menos severas y es así como los comerciantes se han visto afectados ante esta situación.

La incertidumbre de cuánto durará la crisis, ni la forma que podría tener la recuperación, conlleva al análisis de los mecanismos que deben afrontar los comerciantes para su adaptación ante los retos de la COVID-19. Estudios y resultados expuestos en Salles Sainz Grant Thornton S.C. (2020), refieren que las empresas, las PYMES y en general los comerciantes, experimenten dificiles etapas ante la COVID-19, debido a la confluencia entre las constantes crisis que el sistema económico mundial padece, la globalización, la creciente competitividad, el incremento de las demandas de los clientes, las restricciones bancarias y el dinamismo de los mercados.

Cuanto más rápida y contundente sea la respuesta sobre el impacto económico que ocasiona la COVID-19, menores serán los efectos negativos. Algunos de los mecanismos tradicionales de mercado podrían no ser suficientes para enfrentar dicha situación, debido a la interrupción de las actividades productivas y la consiguiente contracción de demanda.

El inesperado viraje de las condiciones del mercado, ante la COVID-19, requiere de un análisis del modelo de negocio existente o del modelo que tienen en cuenta los comerciantes ecuatorianos, de manera particular y en general las empresas y las PYMES. También se requiere del análisis de las estrategias, para a partir del nuevo entorno tomar decisiones, que puedan constituir una valiosa oportunidad, útil para efectuar decisiones estratégicas complejas y que a su vez constituyan mecanismos de adaptación a tener en cuenta ante los retos de la COVID-19.

Ante el referido panorama, las pequeñas y medianas empresas (PYMES) y los comerciantes de forma particular, en Ecuador, no son una excepción, debido a sus características, ya que 
estos son los primeros en recibir con mayor fuerza los impactos que conlleva esta nueva pandemia. Sin embargo, ante estas circunstancias, la planeación (planificación) estratégica, marca la diferencia entre la sostenibilidad y éxito en determinado plazo o el fracaso e ineludible del cierre de operaciones de los comerciantes, por tal motivo, resulta favorable y acucioso pensar y revisar en detalle el negocio y el futuro del mismo.

La planeación (o planificación) estratégica, ante estas situaciones, son de vital importancia y ellas están referidas a un proceso por medio del cual se define la visión (futuro) de los comerciantes y las empresas de forma general y, en atención a éste, el despliegue de determinadas acciones y procedimientos con los cuales es posible alcanzar las proyecciones planificadas (Ruíz, 2019). A pesar de la importancia que reviste el proceso de planificación estratégico, los comerciantes poseen la tendencia de no considerar dicho proceso, lo cual repercute de forma negativa ante cualquier crisis que pueda existir, como es el caso actual de la presencia de la COVID-19.

La planificación estratégica es un ejercicio de formulación y establecimiento de objetivos de carácter prioritario, cuya característica principal es el mapeo de la ruta de acción (estrategia) para alcanzar los objetivos propuestos (Ruíz, 2019). La pandemia que azota a todos los países ha propiciado en Ecuador, un estancamiento económico elevado por cada uno de sus ciudadanos, que requiere de mecanismos para la adaptación de los comerciantes ante los retos de la COVID-19 y su salida o crecimiento económicos post COVID-19.

Ante las actuales condiciones, en Ecuador, se estima que la mitad de todas las empresas del país están corriendo el riesgo de quebrar, y especialmente, las PYMES y las micro empresas; las que constituyen el 99\% de todos los negocios en la nación, y que terminarán siendo las más afectadas (Roa, 2020). La referida estimación, se realiza de acuerdo con el amplio espectro de influencia que la COVID-19, deja como evidencia en el ámbito económico en general.

Basado en el estudio realizado, se evidencian que los efectos del COVID-19, sobre los comerciantes ecuatorianos en particular, se sustentan en la oferta y la demanda de sus comercializaciones, cuya intensidad y profundidad dependerán de las condiciones internas de cada economía y que está en correspondencia con las planificaciones estratégicas que 
hayan trazado, como mecanismo de adaptación ante los retos que impone la COVID-19. Los efectos que a mediano y largo plazo se constatan son:

- Quiebras de empresas.

- Reducción de la inversión privada.

- Menor crecimiento económico.

- Menor integración en cadenas de valor.

- Deterioro de las capacidades productivas y del capital humano.

- Aumento de la pobreza y la pobreza extrema.

Los efectos antes referidos constituyen una agudización de la crisis sanitaria que se ha puesto de manifiesto debido a la fragilidad del sistema globalizado y del modelo de desarrollo subyacente. La ruptura de las cadenas de suministro, la desaceleración del crecimiento mundial y el desempeño de los mercados financieros han mostrado que las economías están expuestas a las vulnerabilidades mundiales.

Para analizar la incidencia que poseen los elementos referidos, sobre los comerciantes ecuatorianos, ante los retos de la COVID-19, se construye una Red Bayesiana, útil para conocer los efectos que a mediano y largo plazo afectarán con mayor énfasis las economías ecuatorianas. De los resultaos obtenidos, se podrán realizar planificaciones estratégicas, que constituirán mecanismos de adaptación ante los retos de la COVID-19 y a su vez dichas planificaciones, contribuyen a minimizar los efectos económicos ocasionados por la pandemia en Ecuador.

Las Redes Bayesianas, son un tipo muy popular de redes probabilísticas (Charles River Analytics Inc., 2004), que proveen información sobre las relaciones de dependencia e independencia condicional existentes entre las variables. La inclusión de las relaciones de independencia en la propia estructura de la red hace de las redes bayesianas una buena herramienta para representar conocimiento de forma compacta pues se reduce el número de parámetros necesarios.

Las técnica de Inteligencia Artificial, ha sido utilizada para realizar tareas de clasificación, en particular tareas de predicción, aun cuando existe una sola variable contenida en bases de datos, cuya variable actúa como un clasificador y a su vez representa el problema que 
se desea predecir, mientras que todas las demás variables son los datos almacenados en la base de datos conformando un conjunto de datos, para tratarlos como casos en diferentes procesos (González, Estrada y Febles, 2018).

En general, una red Bayesiana busca modelar algún fenómeno de interés, al considerar las variables aleatorias involucradas en el problema y la estructura de dependencia existente entre ellas. Así, el objetivo principal consiste en obtener la distribución de probabilidad condicionada de las variables que no son conocidas, principalmente de alguna de ellas definida como variable respuesta, en base a las variables que sí lo son (variables evidenciales). Muchas veces, las variables observables están fijas a priori; sin embargo, otras veces, éstas pueden ir siendo definidas durante el mismo proceso de modelado de la red.

\section{METODOLOGÍA}

Para analizar la incidencia de los efectos del COVID-19, sobre los comerciantes ecuatorianos, se realiza un análisis de sensibilidad a la evidencia en redes bayesianas en aras de conocer los mecanismos de adaptación que deben tener en cuenta, los comerciantes ecuatorianos, de acuerdo con la información que se introduce en la Red Bayesiana, útil para la toma de decisiones para la mejora de las planificaciones estratégicas a tener en cuenta para las comercializaciones futuras. La información sobre los elementos a tener en cuenta para conocer los mecanismos de adaptación ante la COVID-19, varia en correspondencia con las características de los comerciantes, lo que produce cambios en los parámetros de la Red Bayesiana, considerados en la distribución de probabilidad condicionada, o en los valores específicos que se asigne a las variables evidénciales que inciden en los mecanismos de adaptación de los comerciantes ecuatorianos ante los retos de la COVID-19.

La metodología utilizada para el análisis de sensibilidad a la evidencia en Redes Bayesianas para analizar los mecanismos de adaptación de los comerciantes ante los retos de la COVID-19, es la descrita por por Kjaerulff y Madsen (2007), para redes Bayesianas discretas. Esta metodología analiza el valor de la información (value of information analysis), paso a paso, para cuantificar cambios en la distribución de la variable respuesta, al incorporar a la red una variable adicional como evidencia. 
Al respecto se utiliza la entropía, como una medida de incertidumbre de la distribución de la variable respuesta, la metodología se basa en utilizar esta medida para determinar un orden de prioridad al incorporar nuevas evidencias. El procedimiento propuesto consiste en que la variable $\mathrm{X}$, como la variable de interés de la Red Bayesiana discreta (Mecanismo de adaptación), y la variable Y, que caracteriza el conjunto de todas las variables restantes (efectos que a mediano y largo plazo inciden en la economía de los comerciantes ante los retos de la COVID-19) que se caracterizan por ser discretas y están involucradas en la red (inicialmente no observadas), facilitan:

1. Calcular la función valor, definida por $V(X)=-H(X)$.

2. Calcular I $(X ; Y)$ para todo $Y$ no observado.

3. Incorporar a la red como evidencia (e) la variable $Y$ con la que se obtuvo una mayor $\mathrm{I}(X ; Y)$ en 2.

4. Calcular el aumento que se ha producido en $\mathrm{V}(X)=-(H(X)-I(X ; Y=y))=-H$ $(X / Y=y)$.

5. Volver a calcular I $(X ; Y)$ para todo $Y /=e$ incorporado en 3.

Si la variable con mayor I $(X ; Y)$ no es observable, se considera la segunda mayor. Las variables con $\mathrm{I}(X ; Y)=0$ no necesitan ser observadas porque no aportan información a la variable respuesta. Hay que notar que se propone como medida de decisión, la función valor $V(X)=-H(X)$, es decir, el valor negativo de la entropía, en vez de la entropía directamente.

Para justificar esta elección, Kjaerulff y Madsen (2007), proponen considerar la variable de interés $X$ es de tipo binario, con posibles estados verdadero y falso, entonces la distribución de $X$ corresponde a una distribución de Bernoulli de parámetro (p). Luego, Prob $(X=$ verdadero, $X=$ falso $)=(p, 1-p)$ y la entropía máxima se alcanzará en $p=0.5$. Sin embargo, el análisis es factible al obtener la mayor información posible para la distribución de la variable respuesta, más que en disminuir la entropía (aunque en ambos casos se llega al mismo objetivo); por esta razón, se elige trabajar con la función valor, que será mínima cuando $p=0.5$ y máxima en los extremos. Por lo que esta metodología permite generar un orden de prioridad para incorporar variables evidenciales a una red Bayesiana discreta. 


\section{RESULTADOS}

Para analizar los efectos que a mediano y largo plazo inciden en la economía de los comerciantes ante los retos de la COVID-19, se construye la Red Bayesianas (Figura 1), que representa los elementos a tener en cuenta, como mecanismo de adaptación, por parte de los comerciantes ecuatorianos ante la COVID-19 a través de los nodos $(X 1, \ldots X 6)$ donde; $X 1$, se corresponde con las quiebras de empresas, $X 2$, se corresponde con la reducción de la inversión privada, $X 3$, se corresponde con el menor crecimiento económico que se produce, $X 4$, se corresponde con la menor integración en cadenas de valor, $X 5$, se corresponde con el deterioro de las capacidades productivas y del capital humano y $X 6$, se corresponde con el aumento de la pobreza y la pobreza extrema. La variable $X 7$, es considerada la respuesta de interés y se trabajara bajo el supuesto de que inicialmente no hay variables evidénciales determinadas.

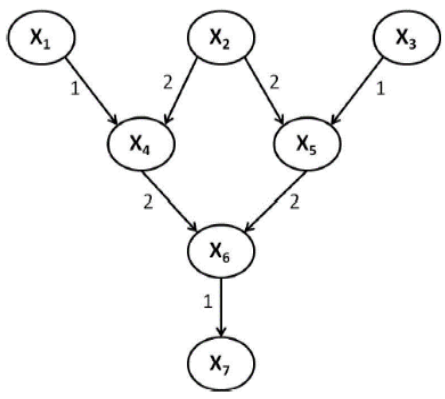

Figura 1. Red Bayesiana sobre los efectos que a mediano y largo plazo inciden en la economía de los comerciantes ante los retos de la COVID-19.

Fuente: elaboración propia.

A partir de la Ecuación 1, y al utilizar la definición de entropía para el caso normal, Ecuación 2 y Normal Multivariante, Ecuación 3, se calculan los pasos 1 y 2 especificados en la metodología propuesta para obtener la entropía diferencial de la variable respuesta $X 7, h(X 7)=3.7165$, y los valores de información mutua para cada una de las variables no evidénciales con la variable respuesta.

$$
\begin{aligned}
I(X ; Y) & =h(X \mid Y) \\
& =h(X \mid Y) \\
& =h(X)+h(Y)-h(X, Y)
\end{aligned}
$$


Cumpliendo que:

$$
\begin{aligned}
& h(X)=\frac{1}{2} \ln \left(2 \pi e \sigma^{2}\right) \\
& h\left(X_{1}, X_{2} \ldots, X_{2}\right)=\frac{1}{2} \ln (2 \pi e)^{n}|\Sigma|
\end{aligned}
$$

Los cálculos se realizaron con el software estadístico de distribución libre $\mathrm{R}$ ( $\mathrm{R}$ Development Core Team, 2013), los resultados obtenidos se muestran en la Tabla 1.

Tabla 1. Análisis de sensibilidad al calcular I $(\mathrm{X} ; \mathrm{Y})$ para todo $\mathrm{Y}$ no observado.

\begin{tabular}{|c|c|c|c|}
\hline $\boldsymbol{Y} \boldsymbol{i}$ & $\boldsymbol{h}\left(\boldsymbol{Y}_{-\boldsymbol{i}}\right)$ & $\boldsymbol{h}\left(\boldsymbol{X} 7, \boldsymbol{Y}_{-\boldsymbol{i}}\right)$ & $\boldsymbol{I}\left(\boldsymbol{X} 7 ; \boldsymbol{Y}_{-\boldsymbol{i}}\right)$ \\
\hline$X 1$ & 1.4190 & 5.1149 & 0.0207 \\
\hline$X 2$ & 1.4990 & 4.6156 & 0.5201 \\
\hline$X 3$ & 1.7656 & 5.4401 & 0.0422 \\
\hline$X 4$ & 2.3149 & 5.4719 & 0.5596 \\
\hline$X 5$ & 2.5703 & 5.5019 & 0.7850 \\
\hline$X 6$ & 3.7064 & 5.4719 & 1.9510 \\
\hline
\end{tabular}

Fuente: elaboración propia.

A partir de la Red Bayesiana (Figura 1), relacionada con los siete efectos que a mediano y largo plazo inciden en la economía de los comerciantes ante los retos de la COVID-19, conectados como se muestra en el grafo de la Figura 1, se obtuvo la distribución conjunta de $X$, correspondiente a una distribución Normal Multivalente con parámetros $\mu$ y $\Sigma$. A partir de estos parámetros, se obtiene que la distribución marginal de la variable $X 7$ (mecanismo de adaptación de los comerciantes ante la COVID-19), corresponde a una distribución Normal $X 7 \sim N(8,99)$.

Los valores demuestran que la distribución asociada posee una alta variabilidad, lo que se ve reflejado en una alta entropía, que denota la existencia de incertidumbre, con los efectos que a mediano y largo plazo inciden en la economía de los comerciantes ante los retos de la COVID-19. Para disminuir dicha incertidumbre, que afecta los comerciantes ecuatorianos, ante la crisis por la COVID-19, se requiere buscar los elementos que no se tienen en cuenta a mediano y largo plazo en el impacto económico de los comerciantes ante los retos de la COVID-19, considerados estos elementos como variables no observables. 
De acuerdo con los resultados mostrados en la Tabla 1, es posible obtener un orden de prioridad para mejorar la entropía de $X 7$ (mecanismo de adaptación de los comerciantes ante la COVID-19). La variable más informativa para $X 7$ (mecanismo de adaptación de los comerciantes ante la COVID-19), es la variable $X 6$ (aumento de la pobreza y la pobreza extrema) y luego, la variable $X 5$ (deterioro de las capacidades productivas y del capital humano).

Para mostrar cómo la elección de la variable a observar afecta en la disminución de la incertidumbre de la variable respuesta $X 7$ (mecanismo de adaptación de los comerciantes ante la COVID-19), se calculó la varianza condicionada y su entropía diferencial condicionada, donde se considera el caso supuesto de que cada una de las variables evidénciales fueron observadas. El resultado obtenido se muestra en la Tabla 2.

Tabla 2. Análisis de sensibilidad de X7 (mecanismo de adaptación de los comerciantes ante la COVID-19) a la evidencia.

\begin{tabular}{|c|c|c|}
\hline $\boldsymbol{Y}-\boldsymbol{i}$ & $\boldsymbol{V a r}(\boldsymbol{X} 7 / \mathbf{Y}-\boldsymbol{i})$ & $\boldsymbol{h}(\boldsymbol{X 7 / Y}-\boldsymbol{i})$ \\
\hline$X 1$ & 96 & 3.6960 \\
\hline$X 2$ & 36 & 3.1967 \\
\hline$X 3$ & 92 & 3.6744 \\
\hline$X 4$ & 32.4 & 3.1565 \\
\hline$X 5$ & 20.7 & 2.9317 \\
\hline$X 6$ & 3 & 1.7656 \\
\hline
\end{tabular}

Fuente: elaboración propia.

Los resultados obtenidos no dependen del valor que toma la variable observada, sino del conjunto de variables evidénciales que se consideren. Basado en que la distribución inicial de $X 7$ (mecanismo de adaptación de los comerciantes ante la COVID-19) es igual a $N$ $(8,99)$, con una entropía diferencial de $h(X 7)=3.7166$; se evidencia que la variable más informativa para $X 7$ (mecanismo de adaptación de los comerciantes ante la COVID-19), es $X 6$ (aumento de la pobreza y la pobreza extrema). Si se incorpora esta variable como evidencia, la varianza de $X 7$ (mecanismo de adaptación de los comerciantes ante la COVID-19) disminuye a 2 y su entropía es igual a 1.77; es decir, sería suficiente observar X6 (aumento de la pobreza y la pobreza extrema) para que el problema quedara resuelto con una alta precisión. 
Por otro lado, se observa que las dos variables que fueron incorporadas como evidencia, X1 (Quiebras de empresas) y X3 (Menor crecimiento económico), no contribuyen individualmente a la reducción de la incertidumbre en $X 7$ (mecanismo de adaptación de los comerciantes ante la COVID-19). Por lo que, para disminuir la incertidumbre, se supone que la variable $X 6$ (aumento de la pobreza y la pobreza extrema) no está disponible, pero que sí se cuenta con información para la variable $X 5$ (deterioro de las capacidades productivas y del capital humano), que es la que produce el siguiente mayor efecto.

Si se incorpora esta variable a la red como evidencia (paso 3, del procedimiento propuesto), entonces la nueva distribución de $X 7 \mid X 5$ es $N(8,20.7)$ y del paso 4 se obtiene una entropía diferencial condicionada $h(X 7 \mid X 5)=2.9317$, que implica una importante reducción de incertidumbre.

De acuerdo con los pasos 5 y 6 respectivamente, del procedimiento propuesto, a través de $X 5$ (deterioro de las capacidades productivas y del capital humano) $\epsilon E$, se calcula la información mutua condicionada del resto de las variables $Y-i$ con $X 7$ (mecanismo de adaptación de los comerciantes ante la COVID-19). Para calcular la distribución Normal Multivariante condicionada por el resto de las variables al propagar $X 5$ (deterioro de las capacidades productivas y del capital humano), con una distribución de $(X 1, X 2, X 3, X 4$, $X 6, X 7$ I X5) y luego hacer los cálculos, en forma análoga a los realizados para la Tabla 1, los resultados que se obtienen se muestran en la Tabla 3.

Tabla 3. Análisis de sensibilidad, X7 (mecanismo de adaptación de los comerciantes ante la COVID-19), condicionado en X5 (Deterioro de las capacidades productivas y del capital humano).

\begin{tabular}{|c|c|c|c|}
\hline $\boldsymbol{Y}-\boldsymbol{i}$ & $\boldsymbol{h}(\boldsymbol{Y}-\boldsymbol{i} \mid \boldsymbol{X} \mathbf{5})$ & $\boldsymbol{h}(\boldsymbol{X} \mathbf{7}, \boldsymbol{Y}-\boldsymbol{i} \mid \boldsymbol{X} \mathbf{5})$ & $\boldsymbol{I}(\boldsymbol{X} \mathbf{7} ; \boldsymbol{Y}-\boldsymbol{i} \mid \mathbf{X 5})$ \\
\hline$X 1$ & 1.4190 & 4.2427 & 0.1080 \\
\hline 22 & 1.1636 & 3.7815 & 0.3138 \\
\hline 33 & 1.6540 & 4.5452 & 0.0405 \\
\hline 4 & 2.1598 & 4.1280 & 0.9634 \\
\hline 66 & 2.8806 & 4.6461 & 1.1662 \\
\hline
\end{tabular}

Fuente: elaboración propia.

Los resultados mostrados en la Tabla 3, demuestran que la variable X6 (Aumento de la pobreza y la pobreza extrema) es la que permite disminuir en forma más significativa la incertidumbre de $X 7$ (mecanismo de adaptación de los comerciantes ante la COVID-19), pero como se ha considerado que esta variable no puede ser observada, se incorporará 
como evidencia la segunda variable más informativa para $X 7$ (mecanismo de adaptación de los comerciantes ante la COVID-19), que es la variable $X 4$ (Menor integración en cadenas de valor). Para ello se retorna al paso 3, del procedimiento propuesto, después de propagar la variable evidencial $X 4$, y se obtiene que la nueva $\operatorname{Var}(X 7 \mid X 5, X 4)=3$, lo que significa que se ha podido obtener un resultado con una alta precisión.

Finalmente, la entropía diferencial condicionada que se ha obtenido para $X 7$ I $X 5$, $X 4$ es $h$ $(X 7 \mid X 5, X 4)=1.969$, en la Tabla 4 , se muestran los nuevos valores de entropía e información mutua de las variables que catalogadas como no evidénciales. Se observa que la entropía diferencial de la variable respuesta $X 7$ (mecanismo de adaptación de los comerciantes ante la COVID-19), ha disminuido notoriamente y que ya no existen variables no evidénciales que aporten información relevante, por otra parte, es de destacar que las variables $X 1$ (Quiebras de empresas), X2 (Reducción de la inversión privada) y X3 (Menor crecimiento económico), tienen valores de información mutua con $X 7$ (mecanismo de adaptación de los comerciantes ante la COVID-19) de cero, lo que significa que ellas son independientes de $X 7$ (mecanismo de adaptación de los comerciantes ante la COVID-19), dado X4 (Menor integración en cadenas de valor) y $X 5$ (Deterioro de las capacidades productivas y del capital humano), como se muestra en el DAG, de la Figura 1.

Tabla 4. Análisis de Sensibilidad, condicionado en X4 (Menor integración en cadenas de valor) y X5 (Deterioro de las capacidades productivas y del capital humano).

\begin{tabular}{|c|c|c|c|}
\hline$Y-i$ & $h(Y-i \mid X 5, X 4)$ & $h(X 7, Y-i \mid X 5, X 4)$ & $I(X 7 ; Y-i \mid X 5, X 4)$ \\
\hline$X 1$ & 1.2884 & 3.2566 & 0 \\
\hline$X 2$ & 0.7644 & 2.7326 & 0 \\
\hline$X 3$ & 1.6048 & 3.5730 & 0 \\
\hline$X 6$ & 1.4190 & 3.1845 & 0.2028 \\
\hline
\end{tabular}

Fuente: elaboración propia.

\section{CONCLUSIONES}

En el presente trabajo se realizó un abordaje de los mecanismos de adaptación de los comerciantes ecuatorianos, ante los retos de la COVID-19. Se evidenciaron los efectos que a mediano y largo plazo se deben tener en cuenta para crear los mecanismos de adaptación en aras de una mejora económica y de comercialización. 
De acuerdo con los elementos identificados se construyó una Red Bayesiana donde se analizaron los efectos de mayor peso, que a mediano y largo plazo provoca la COVID-19, para los comerciantes ecuatorianos de forma particular y para las empresas de forma general. Para ello se realizó un análisis de sensibilidad a la evidencia de los elementos identificados con el fin de proyectar los mecanismos adaptación de los comerciantes ecuatorianos, ante los retos de la COVID-19, el cual se apoyó en los resultados obtenidos de los cálculos realizados a través de la Red Bayesiana, donde se interrelacionaron los efectos que a mediano y largo plazo provoca la COVID-19, desde la perspectiva de desarrollo económico.

Se constató que el resultado obtenido contribuye al apoyo a la toma de decisiones, para que los comerciantes creen sus propias proyecciones estratégicas en base a los efectos que a mediano y largo plazo provoca la COVID-19, considerándose dichas proyecciones, los mecanismos de adaptación a tener en cuenta ante los retos que ha impuesto esta enfermedad.

El análisis de sensibilidad a la evidencia en Redes Bayesianas realizado constituye una metodología útil para proyectar las planeaciones estratégicas, como el ejercicio exitoso de cualquier organización ante retos como los que ha impuesto la COVID-19. Las proyecciones estratégicas de los comerciantes ecuatorianos es un proceso de apoyo es la base que constituye el mecanismo de adaptación a tener en cuenta, ante los retos de la COVID-19 y significa la vía sobre la cual estarán encaminadas las funciones de los comerciantes y las empresas.

\section{REFERENCIAS BIBLIOGRÁFICAS}

Gharles River Analytics Inc. (2004). About Bayesian Belief Networks. https://cs.ipb. ac.id/ yeni/files/Introduction \%20to\%20Bayesian\%20Network.pdf

González, N., Estrada, V., y Febles, A. (2018). Estudio y selección de las técnicas de Inteligencia Artificial para el diagnóstico de enfermedades. Revista Ciencias Médicas de Pinar del Río, 22(3), 534-544. www.revcmpinar.sld.cu/index.php/publicaciones/ article/view/3465

Kjaerulff, U., y Madsen, A. (2007). Probabilistics Networks for Practitioners. A Guide to Construction and Analysis of Bayesian Networks and Influence Diagrams. Springer. 
R Development Gore Team. (2013). R: A Language and Environment for Statistical Computing. $R$ Core Team Foundation for Statistical Computing. http:/ / www.r-project.org

Roa, S. (2020). Las más afectadas. GK. https://gk.city/2020/06/28/pymes-crisis-covid19ecuador/

Ruíz, M. (2019, 11 de abril). Plan Estratégico de una empresa PyME: ¿Qué es, cómo se hace?, paso a paso. https://milagrosruizbarroeta.com/plan-estrategico-empresa-pyme-que-escomo-se-hace/

Salles Sainz Grant Thornton S.G. (2020, 19 de marzo). Crecer a pesar del COVID-19. https://www.grantthornton.mx/prensa/marzo_20202/Crecer_a_pesar_del_ COVID19/ 